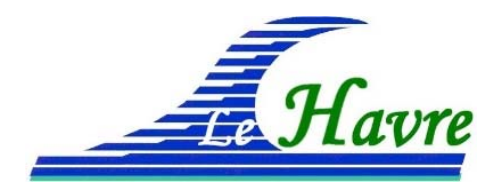
XVI èmes Journées Nationales Génie Côtier - Génie Civil
Le Havre, 2020

DOI:10.5150/jngcgc.2020.069 C Editions Paralia CFL

disponible en ligne - http://www.paralia.fr - available online

\title{
Modélisation numérique des rejets bactériologiques sur l'estuaire de la Laïta
}

\section{Jérémy DUGOR ${ }^{1}$, Vanessa THORIN ${ }^{2}$, Romain SUAUDEAU ${ }^{2}$, Julien BAILLS ${ }^{1}$, Rémi BUR ${ }^{1}$, Didier RIHOUEY ${ }^{1}$, Hervé BIZIEN ${ }^{1}$}

1. CASAGEC INGENIERIE, 1 rue Jean Torlais, 17000 La Rochelle, France. contact@casagec.fr

2. SMEIL-Syndicat Mixte Ellé Isole Laïta, 1 rue Andreï Sakharov, 29394 Quimperlé, France.

smeil@quimperle-co.bzh

\section{Résumé :}

L'estuaire de la Laïta se situe sur la côte de Bretagne Sud. Dégradée depuis longtemps par différentes sources de pollution, la qualité bactériologique de la Laïta s'est améliorée ces dernières années, confirmée par une récente autorisation pour les activités de conchyliculture à l'aval de l'estuaire. Afin d'améliorer les connaissances du système et d'identifier les leviers d'amélioration de la qualité de l'eau, un modèle numérique tridimensionnel (Télémac 3D) a été mis en place. De nombreuses données ont permis de calibrer le modèle numérique.

Dans le cadre de cette étude, une formulation (ERICHSEN et al., 2006) a été implémentée dans le modèle numérique qui permet de calculer le coefficient de dégradation $(\mathrm{k})$ des bactéries en fonction des paramètres principaux ayant une influence sur la mortalité des germes tels que la salinité, la température, la profondeur et l'intensité de la lumière.

Pour les scénarios de calibration, les flux de bactéries imposés au modèle sont issus pour la plupart de campagnes de mesures ou d'analyses paramétriques. Des points de mesures à l'aval de l'estuaire, proches des zones d'activités conchylicoles, sont utilisés pour valider le modèle selon les conditions fluvio-maritimes. Plusieurs scénarios sont étudiés afin d'analyser l'impact de chaque source de contamination. Des scénarios de pollutions accidentelles sont également étudiés.

Cet article présente la calibration du modèle, la hiérarchisation des flux bactériologiques, une brève analyse sur les impacts et les éventuelles solutions.

\section{Mots clés :}

Loi de décroissance des bactéries fécales, Modélisation estuarienne 3D

\section{Introduction}

\subsection{Caractéristiques de l'estuaire de la Laïta}

La Laïta est un fleuve côtier, situé à la limite des départements du Finistère et du Morbihan (figure 1). La Laïta est formée par la confluence de l'Isole et de l'Ellé au centre- 


\section{Thème 6 - Gestion durable des zones littorales et estuariennes}

ville de Quimperlé, et s'étend sur $17 \mathrm{~km}$ jusqu'à son embouchure. L'Isole et l'Ellé drainent un bassin versant d'une superficie totale de $829 \mathrm{~km}^{2}$. La Laïta reçoit les eaux des deux cours d'eau précédents en drainant son propre bassin versant d'une superficie de 88 $\mathrm{km}^{2}$ avec les principaux cours d'eau suivants : le Dourdu (RD - rive droite), le Frout (RD), le ruisseau de Kerhyuel (RG - rive gauche), de Saint-Michel (RG), et le Quinquis (RD). L'estuaire est soumis à un climat océanique, une variation marquée des débits est observable entre l'été $\left(\sim 3 \mathrm{~m}^{3} / \mathrm{s}\right)$ et la période hivernale $\left(\sim 30 \mathrm{~m}^{3} / \mathrm{s}\right)$. Les débits peuvent dépasser $500 \mathrm{~m}^{3} / \mathrm{s}$ pour une crue centennale sur la Laïta. Le tableau 1 fournit les débits caractéristiques des principaux cours d'eau.
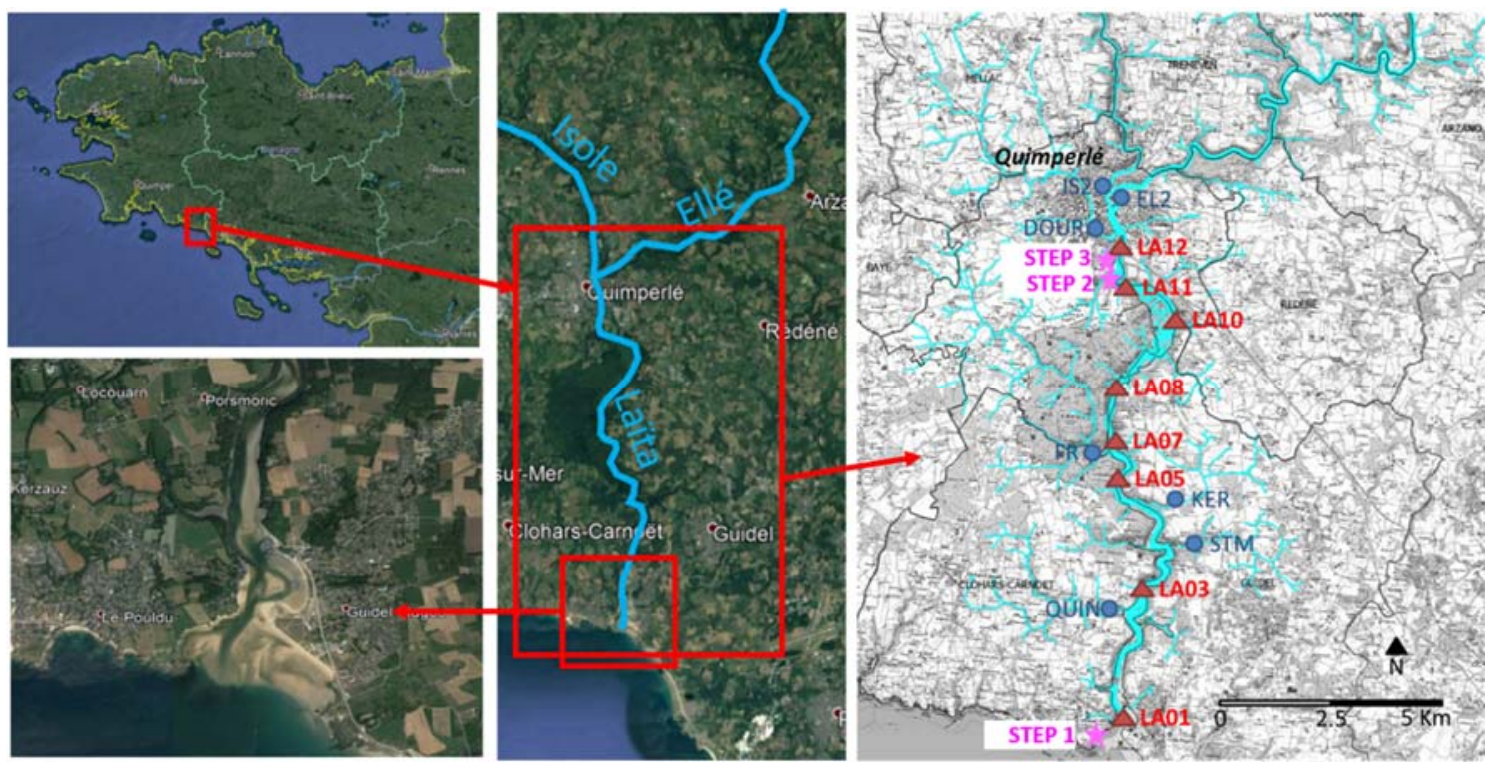

Figure 1. A gauche, localisation de la Laïta. A droite, emplacement des points de surveillance. Points bleus : mesures aux exutoires; triangles rouges : réseaux de suivis ; étoiles roses : rejets des stations d'épuration des eaux usées (STEP) numérotés dans la Laïta.

Tableau 1. Débits de l'Ellé, l'Isole et la Laïta de 1969 à 2018, source : Banque Hydro.

\begin{tabular}{cccccc}
\hline Rivière & $\begin{array}{c}\text { Superficie } \\
\text { Bassin versant }\end{array}$ & $\boldsymbol{Q}_{\text {étiage }}$ & $\boldsymbol{Q}_{\text {module }}$ & $\boldsymbol{Q}_{\text {crue10 }}$ & $\boldsymbol{Q}_{\text {crue10o }}$ \\
\hline Ellé à Arzano & $603 \mathrm{~km}^{2}$ & $1 \mathrm{~m}^{3} / \mathrm{s}$ & $9,5 \mathrm{~m}^{3} / \mathrm{s}$ & $110 \mathrm{~m}^{3} / \mathrm{s}$ & $330 \mathrm{~m}^{3} / \mathrm{s}$ \\
Isole à Quimperlé & $226 \mathrm{~km}^{2}$ & $0,5 \mathrm{~m}^{3} / \mathrm{s}$ & $4,3 \mathrm{~m}^{3} / \mathrm{s}$ & $51 \mathrm{~m}^{3} / \mathrm{s}$ & $200 \mathrm{~m}^{3} / \mathrm{s}$ \\
Laita à Quimperlé & $832 \mathrm{~km}^{2}$ & $2 \mathrm{~m}^{3} / \mathrm{s}$ & $13,9 \mathrm{~m}^{3} / \mathrm{s}$ & $160 \mathrm{~m}^{3} / \mathrm{s}$ & $>500 \mathrm{~m}^{3} / \mathrm{s}$ \\
\hline
\end{tabular}

Le littoral est soumis à une marée de type semi-diurne avec un marnage de $2 \mathrm{~m}$ en morteeau, de 4,1 m en vive-eau et jusqu'à 5,5 m pour les plus grandes marées. La limite d'action de la marée dynamique se situe à Quimperlé, dans l'Ellé à environ $950 \mathrm{~m}$ en amont de la confluence, au niveau d'un seuil. La marée ne remonte quasiment pas dans l'Isole 


\section{XVI'̀mes Journées Nationales Génie Côtier - Génie Civil \\ Le Havre 2020}

(présence d'un clapet mobile anti-crue). La position du front de salinité peut remonter entre 4 et $8 \mathrm{~km}$ en fonction du coefficient de marée et du débit fluvial. Durant les fortes crues, les masses d'eau salées sont bloquées à proximité de l'embouchure (DUGOR et al., 2019).

\subsection{Principales sources de contamination}

Dans cette étude, seule la bactérie Escherichia Coli (E. Coli) est étudiée. Plusieurs sources de contamination ont été identifiées à l'échelle du seul sous bassin-versant (BV) de la Laïta $\left(88 \mathrm{~km}^{2}\right)$. En effet, après une approche globale menée à l'échelle du BV EIL (ElléIsole-Laïta), très vaste, les acteurs ont volontairement décidé de prioriser dans un premier temps l'approche à l'échelle du sous BV de l'estuaire, pour assurer une efficacité optimale des mesures sur un petit territoire. Premièrement, $65 \%$ de la population est connectée à un réseau d'assainissement collectif. Ces réseaux sont traités par 3 stations de traitement des eaux usées (STEP) rejetant directement les effluents dans l'estuaire (numérotées sur la figure 1). Deux rejets sont localisés directement en aval de Quimperlé. Le troisième situé à l'embouchure, est équipé de bioréacteurs à membranes (BRM) permettant une concentration limitée en sortie (250 UFC/100 ml). Par ailleurs, 1700 assainissements non-collectifs ont été recensés dans les sous bassins versants directs de la Laïta. Sur le volet agricole, 70 exploitations ont été listées dans le secteur d'étude, représentant 3785 hectares de surface agricole utile. Des diagnostics permettant d'évaluer la conformité des installations ont été réalisés sur une vingtaine d'exploitations. Par ailleurs une cinquantaine d'abreuvements directs et de passages à gués sont recensés, auxquels s'ajoute un linéaire supérieur à $2 \mathrm{~km}$ de linéaire piétiné. Plusieurs autres sources de contaminations peuvent être listées comme le stockage des bactéries dans les sédiments, les activités de plaisance, l'avifaune...

\subsection{Les enjeux liés à la qualité de l'eau de l'estuaire}

Sur la Laïta, les principaux enjeux de qualité d'eau concernent la baignade (en aval), la mytiliculture (en aval) et les activités nautiques comme le kayak. Différentes normes réglementaires encadrent les concentrations admissibles pour ces pratiques. Un dépassement de ces normes peut amener à des Arrêtés d'interdiction de ces activités.

\section{Suivis bactériologiques}

Afin de contrôler la qualité des eaux de la Laïta, plusieurs réseaux de suivis ont été mis en place depuis plus de 30 ans. Pour cette étude, seuls les suivis sur les principaux affluents ont été utilisés. Il y a aussi des contrôles effectués sur les rejets des STEP (figure 1). Le tableau 2 donne les flux bactériologiques des principales sources de contamination de la Laïta pour les périodes humides, sèches et globales. Les résultats moyens et percentiles sont différenciés. Les mesures (concentrations d'E. Coli et débits) sont effectuées en aval de chaque affluent. Une "période sèche" correspond à une période sans 


\section{Thème 6 - Gestion durable des zones littorales et estuariennes}

pluie dans le bassin versant au cours des 10 jours précédents et une "période humide" lorsqu'il y a une augmentation globale du débit des rivières en raison de la pluie au cours des 24 dernières heures $(>10 \mathrm{~mm})$. La figure 2 illustre les sources de contamination et aussi des points de contrôle le long de la rivière (LA01 à LA12).

Tableau 2. Principaux flux de contaminants déterminés grâce aux réseaux de suivi.

L'échelle de couleur indiquée dans le tableau est affectée en fonction des flux (UFC/s).

\begin{tabular}{|c|c|c|c|c|c|c|c|c|}
\hline \multirow[b]{3}{*}{ Flux (UFC/s) } & \multirow{2}{*}{\multicolumn{3}{|c|}{ Moyenne géométrique }} & \multirow{2}{*}{\multicolumn{3}{|c|}{ Centile 95}} & \multirow{3}{*}{$\begin{array}{l}\text { Rang flux } \\
\text { moyens }\end{array}$} & \multirow{3}{*}{$\begin{array}{c}\text { Rang flux } \\
\text { pics }\end{array}$} \\
\hline & & & & & & & & \\
\hline & Global & Pluie & $\begin{array}{c}\text { Suivi } \\
\text { calendaire }\end{array}$ & Global & Pluie & $\begin{array}{c}\text { Suivi } \\
\text { calendaire }\end{array}$ & & \\
\hline Isole (IS2) & $1,70 E+07$ & $7,63 E+07$ & $3,36 E+06$ & $1,97 E+09$ & $4,28 E+09$ & $8,80 E+07$ & 2 & 2 \\
\hline Ellé (EL2) & $2,98 E+07$ & $1,19 E+08$ & $6,18 E+06$ & $2,60 E+09$ & $9,21 E+09$ & $8,02 E+07$ & 1 & 1 \\
\hline Dourdu (DOUR) & $1,17 E+07$ & $2,12 E+07$ & $5,88 E+06$ & $5,77 E+08$ & $6,51 E+08$ & $5,48 E+08$ & 3 & 4 \\
\hline STEP de Bigar & $1,06 E+07$ & / & / & $2,67 E+08$ & / & / & 4 & 5 \\
\hline STEP de Quimperlé & $4,83 E+06$ & / & / & $5,47 E+07$ & / & / & 5 & 7 \\
\hline Frout (FR) & $5,26 E+05$ & $6,70 E+06$ & $4,23 E+04$ & $7,42 E+08$ & $2,04 E+09$ & $6,90 E+07$ & 7 & 3 \\
\hline Keryhuel (KER) & $4,89 E+05$ & $1,80 E+06$ & $4,54 E+04$ & $2,27 E+07$ & $2,49 E+07$ & $3,88 E+05$ & 8 & 9 \\
\hline St Michel (STM) & $3,88 E+05$ & $7,39 E+05$ & $1,32 E+05$ & $2,46 E+07$ & $2,57 E+07$ & $5,04 E+05$ & 9 & 8 \\
\hline Quinquis (QUIN) & $6,49 E+05$ & $1,57 E+06$ & $1,81 E+05$ & $5,96 E+07$ & $9,72 E+07$ & $5,05 E+06$ & 6 & 6 \\
\hline STEP de Clohars & $2,82 E+03$ & / & I & $3,51 E+04$ & / & / & 10 & 10 \\
\hline
\end{tabular}

\section{Mise en place du modèle numérique}

\subsection{Maillage de calcul}

L'emprise du maillage permet de bien représenter les processus océaniques et fluviaux. La grille est composée de 31500 nœuds et d'environ 59000 triangles. Les dimensions des mailles vont de $250 \mathrm{~m}$ en mer à $3 \mathrm{~m}$ autour des piles du pont de Laïta. Il a été construit pour obtenir un temps de calcul raisonnable sur un ordinateur de base, (ACRI, 2018). La discrétisation tridimensionnelle est représentée par 5 plans sigmas horizontaux dont la distribution est représentée le long du thalweg de la Laïta sur la figure 2 ci-après.

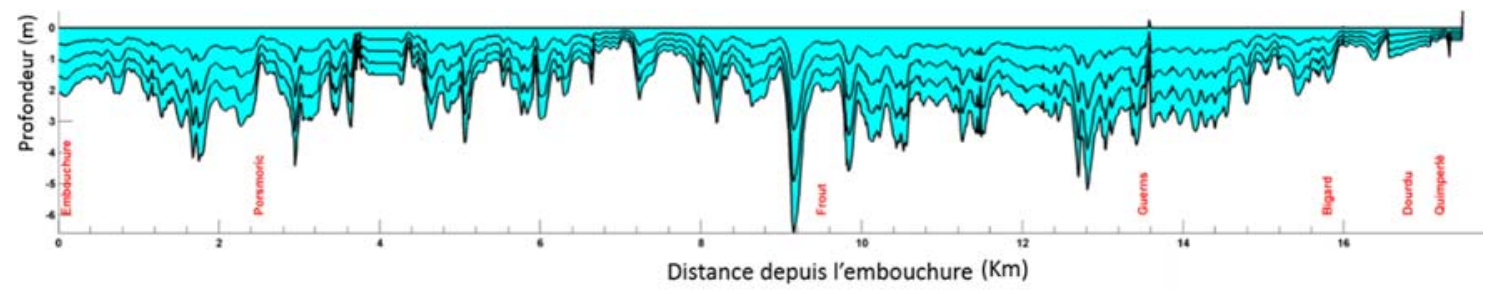

Figure 2. Discrétisation verticale (sigma) utilisée le long du thalweg de la Laïta.

\subsection{Loi de décroissance des bactéries}

La dégradation générale des matières fécales, comme E. Coli, est supposée suivre un modèle exponentiel général de premier ordre (CHICK \& MARTIN, 1908). 


\section{XVIèmes Journées Nationales Génie Côtier - Génie Civil \\ Le Havre 2020}

$C(t)=C(t=0) * e^{(-k * t)}$

où $C$ est la concentration de l'organisme, $t$ le temps et $k$ le coefficient de vitesse de désintégration à $20^{\circ} \mathrm{C}$.

Le coefficient du taux de dégradation peut être décrit de plusieurs manières. La méthode de calcul $k$ est basée sur des informations concernant la température de l'eau, l'intensité lumineuse et la salinité. Dans ce cas, on suppose un apport d'oxygène infini (ERICHSEN et al., 2006 ; SELMEUS, 2018).

Le paramètre de désintégration $k$ dépend des conditions de luminosité et est décrit dans l'équation ci-dessous.

$k=K_{m}+K_{L} * I_{z}$

où $K_{m}$ est la contribution de décroissance en condition d'obscurité, $K_{L}$ est la contribution de décroissance quand les conditions sont lumineuses, $I_{z}$ est l'intensité lumineuse à la profondeur $z$. La contribution de la désintégration, lorsque les conditions sont sombres, est calculée à l'aide de l'équation ci-dessous.

$K_{m}=a_{T} * T-k_{m 0}$

où $a_{T}$ est la constante de dépendance à la température, $T$ est la température réelle de l'eau et $k_{m 0}$ est la constante de vitesse initiale de désintégration des coliformes. Elle n'est valide qu'entre 4 et $24{ }^{\circ} \mathrm{C}$.

$K_{L}=S_{m} * \frac{\left(b_{T} * T+K_{L 0}\right)}{a * S_{m}-\left(\frac{1}{a}\right) * S}$

où $S$ est la salinité réelle, $S_{m}$ est la constante de salinité de référence, a est la correction de la constante de salinité, $b_{T}$ est la constante de dépendance à la température pour la réaction légère et $K_{L 0}$ est le taux de désintégration coliforme initial pour la réaction légère. Cette équation n'est valable qu'entre 12 et $34{ }^{\circ} \mathrm{C}$ (ERICHSEN et al., 2006).

L'intensité lumineuse à la profondeur $z, I_{z}$ peut être décrite par la loi de Lambert-Beer.

$I_{z}=I_{0} * e^{-\mu * z}$

où $I_{0}$ est l'intensité lumineuse à la surface de l'eau, $\mu$ est le coefficient d'extinction et $z$ la profondeur de la contamination. Le coefficient d'extinction peut être déterminé à partir de la profondeur de Secchi, $S D$. Pour la détermination de $\mu$, on suppose que la profondeur de Secchi correspond à la profondeur à laquelle 15\% du rayonnement lumineux persiste. Ce rapport peut toutefois varier d'environ $10 \%$ à $25 \%$ selon la zone.

$\mu=-\ln \left(\frac{0.15}{S D}\right)$

En résumé, la dégradation d'E. coli peut être décrite dans une seule équation :

$\left.C_{E . \text { coli }}=C_{E . \text { coli } 0} * e^{\left(-\left(\left(a_{T} * T-k_{m 0}\right)+\left(S_{m} * \frac{\left(b_{T} * T+K_{L 0}\right.}{a * S_{m}-\left(\frac{1}{a}\right) * S}\right) *\left(I_{0} * e^{\left.-\left(-\ln \left(\frac{0.15}{S D}\right)\right) * z\right)}\right) * t\right.\right.}\right)$ 


\section{Thème 6 - Gestion durable des zones littorales et estuariennes}

Cette équation permet de modéliser les processus de décomposition des bactéries dans le temps et l'espace en prenant en compte les facteurs externes tels que la salinité, l'afflux de lumière et la température de l'eau.

\subsection{Forçages}

Sur la partie océanique, le modèle est d'abord contraint avec le modèle global de marée TPXO (EGBERT et al., 2002) intégrant la variation des courants et des niveaux d'eau liés à la marée astronomique. Sur la partie terrestre, les débits des principaux affluents de la Laïta sont pris en compte avec une concentration en E. Coli correspondante. Les rejets d'effluents provenant des STEP sont également inclus dans la modélisation. Ils sont représentés par une concentration et un écoulement caractéristique. Les simulations sont généralement configurées sans concentration bactérienne en condition initiale. L'eau contaminée se mélange à l'eau de mer en quelques cycles de marée.

\subsection{Validation du modèle hydraulique}

Diverses conditions hydrauliques ont été observées en février 2017 avec un ADCP (Acoustic Doppler Current Profiler) mouillé en aval de Quimperlé (ACRI, 2018). Des cycles des marées de vive-eau et de morte eau avec une période de faibles débits $\left(15 \mathrm{~m}^{3} / \mathrm{s}\right)$ et une période de crue $\left(\mathrm{Q}>50 \mathrm{~m}^{3} / \mathrm{s}\right)$ ont pu être enregistrés. Cette campagne a été très utile pour calibrer le modèle avec différentes conditions hydrauliques. Des mesures de salinité ont permis également de vérifier la bonne représentativité du modèle de dispersion saline.

\subsection{Calibration du modèle de dispersion bactériologique}

Quatre levés ont été sélectionnés pour représenter différentes conditions hydrauliques et marémotrices (voir tableau 3) et pour lesquels les principales mesures en amont sont disponibles. Pour chaque source de contaminant, les flux de rejets ( $\mathrm{Q}$ et concentration en E. Coli) ont été imposés au modèle selon les mesures. Pour les STEP 1 et 2, les rejets et les concentrations ont été estimés avec la moyenne mensuelle en raison d'un manque de mesures dans le cadre de cette étude. Pour la STEP 3, les valeurs de concentration sont relevées de manière journalière. Elles peuvent être très élevées. Pour les affluents ne disposant pas de données, il a été décidé de ne pas les inclure car ils ne représentent qu'une petite partie des sources de contaminants. La figure 3 montre les résultats de modèle de concentration en E. Coli sur une coupe longitudinale de Quimperlé (à droite) jusqu'à l'embouchure (à gauche) à différents moments d'un cycle de marée (campagne \#1).

Les résultats du modèle montrent la dispersion de la contamination en fonction de la marée, avec des concentrations plus élevées en amont, diminuant vers l'embouchure de la rivière. La contribution du débit bactérien du Frout peut être observée (LA07) avec son panache allant vers Quimperlé pendant l'inversion des courants à marée haute. 


\section{XVI'̀mes Journées Nationales Génie Côtier - Génie Civil \\ Le Havre 2020}

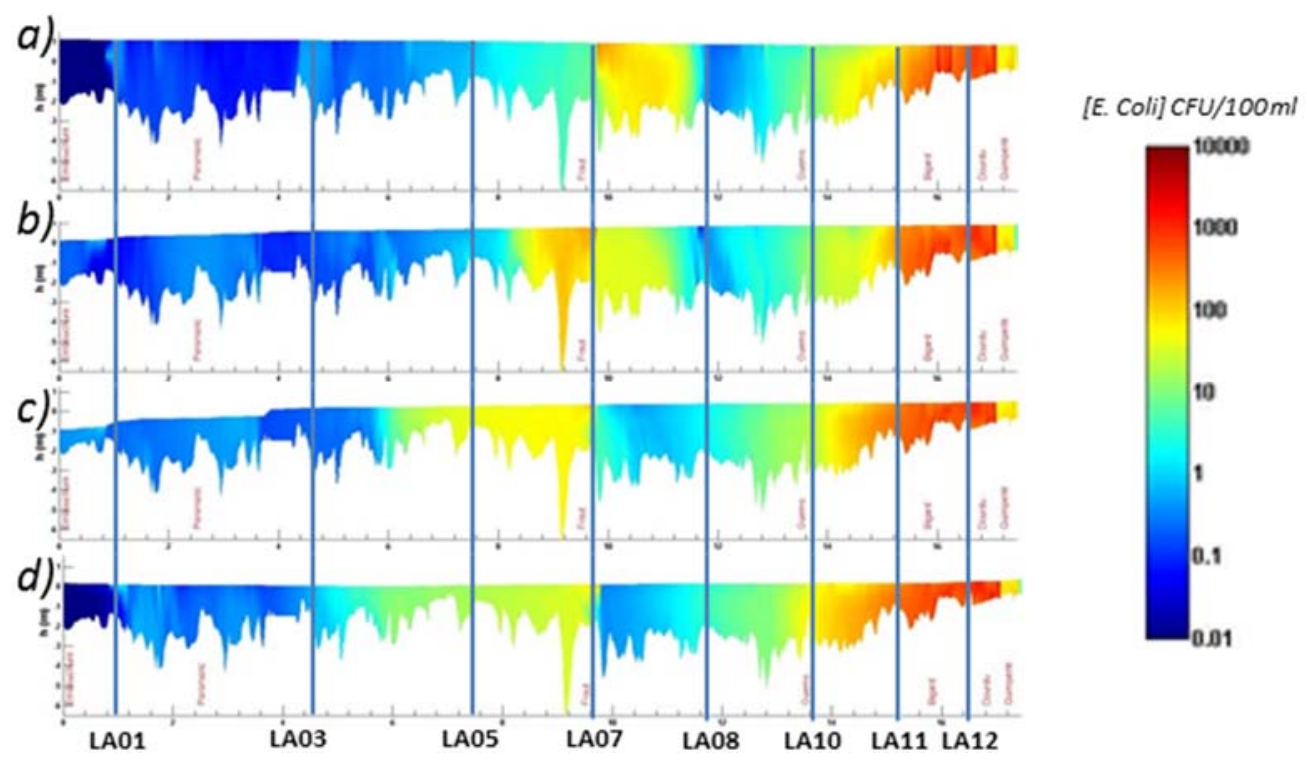

Figure 3. Résultats fournis par le modèle pour les concentrations en E Coli sur un profil longitudinal de la Laïta de Quimperlé (à droite) à l'embouchure (à gauche) pour a) la pleine mer (PM), b) PM-3h, c) la basse mer (BM) et d) $B M+3 h$.

Tableau 3. Principales caractéristiques des campagnes de mesures de calibration. Les concentrations en E. Coli sont exprimés en UFC/100ml.

\begin{tabular}{|c|c|c|c|c|c|}
\hline Campagn & & $1-21 / 07 / 2011$ & $2-09 / 04 / 2015$ & $3-26 / 04 / 2012$ & $4-15 / 12 / 2014$ \\
\hline Coefficie & le marée & 64 & 75 & 65 & 38 \\
\hline Q Laïta (I & & 2,95 & 9,72 & 63 & 22,4 \\
\hline Condition & & Eté - sec & Hiver-sec & Hiver - humide & Hiver - humide \\
\hline Ellé & $Q$ & 1.9 & 6.67 & 42 & 15.2 \\
\hline & [E. Coli] & 78 & 38 & 2000 & 403 \\
\hline Isole & $Q$ & 1.05 & 3.05 & 21 & 7.2 \\
\hline & [E. Coli] & 1000 & 1000 & 8900 & 500 \\
\hline Dourdu & $Q$ & 0.07 & 0.1 & 0.5 & 0.5 \\
\hline & [E. Coli] & 14000 & 5840 & 2700 & 2990 \\
\hline Frout & & 0.015 & 0.04 & 0.25 & 0.25 \\
\hline & [E. Coli] & 510 & 1749 & 8300 & 457 \\
\hline STEP 3 & $Q$ & 0.04 & 0.04 & 0.04 & 0.04 \\
\hline & [E. Coli] & 1700000 & 1015000 & 620000 & 920 \\
\hline STEP 2 & $Q$ & 0.06 & 0.06 & 0.06 & 0.06 \\
\hline & [E. Coli] & 32000 & 32000 & 32000 & 32000 \\
\hline STEP 1 & & 0.02 & 0.02 & 0.02 & 0.02 \\
\hline & [E. Coli] & 17 & 17 & 17 & 17 \\
\hline
\end{tabular}

Dans toutes les campagnes, une diminution générale de la concentration bactérienne peut être observée en aval de la rivière, car la salinité est plus élevée.

Ainsi, la calibration du modèle a été principalement axée sur le paramètre $a_{T}$ de l'équation 3. Le réglage de ce paramètre avec les quatre simulations basées sur les campagnes de mesures exposées ci-dessus donne des résultats satisfaisants (tableau 4). 


\section{Thème 6 - Gestion durable des zones littorales et estuariennes}

Tableau 4. Comparaison des concentrations en E. Coli entre les observations et les résultats de modélisation (UFC/100ml - échelle de couleur adaptée).

\begin{tabular}{cccccccccc}
\hline Campagne \# & LA12 & LA11 & LA10 & LA08 & LA07 & LA05 & LA03 & LA01 \\
\hline \multirow{2}{*}{$\mathbf{1}$} & obs. & 8300 & 6600 & 6100 & 1400 & 510 & 460 & 46 & 15 \\
& Model & $\mathbf{2 0 0 0}$ & $\mathbf{1 1 0 0 0}$ & $\mathbf{2 7 6 0}$ & $\mathbf{7 5}$ & $\mathbf{2 0 0}$ & $\mathbf{1 2 0}$ & $\mathbf{3}$ & $\mathbf{1}$ \\
\multirow{2}{*}{$\mathbf{2}$} & obs. & 728 & 1494 & 1104 & 2779 & 1749 & 858 & 94 & 15 \\
& Model & $\mathbf{7 8 9}$ & $\mathbf{3 4 0 0}$ & $\mathbf{2 3 5 0}$ & $\mathbf{1 4 5 0}$ & $\mathbf{1 1 3 0}$ & $\mathbf{6 5 0}$ & $\mathbf{8 1}$ & $\mathbf{2 0}$ \\
\multirow{2}{*}{$\mathbf{3}$} & obs. & 8300 & 11000 & 6100 & 7700 & 8300 & 11000 & 8900 & 6100 \\
& Model & $\mathbf{3 8 8 0}$ & $\mathbf{4 1 8 0}$ & $\mathbf{3 9 0 0}$ & $\mathbf{3 6 1 0}$ & $\mathbf{4 8 3 0}$ & $\mathbf{3 4 3 0}$ & $\mathbf{2 9 1 0}$ & $\mathbf{2 5 2 0}$ \\
\multirow{4}{*}{$\mathbf{4}$} & obs. & 465 & 480 & 434 & 563 & 457 & 633 & 919 & 415 \\
& Model & $\mathbf{6 5 0}$ & $\mathbf{6 7 0}$ & $\mathbf{5 9 0}$ & $\mathbf{4 7 0}$ & $\mathbf{4 1 0}$ & $\mathbf{3 5 0}$ & $\mathbf{2 1 0}$ & $\mathbf{1 3 0}$ \\
\hline
\end{tabular}

Les comparaisons globales des résultats du modèle avec les mesures in situ indiquent une bonne représentativité du modèle. Cependant, la campagne \#3 et quelques points de comparaison donnent des différences significatives. Pour la campagne \#3, le modèle sous-estime les concentrations d'un facteur 3 le long de l'estuaire. Un débit important est observé lors de cette campagne, ainsi le protocole de mesure pourrait être discutable.

Autour de LA12, LA11 et LA10, les différences peuvent également atteindre un facteur de 3 au maximum. Elles peuvent être dues par l'estimation du flux bactérien des STEP $1 \& 2$. Il y a aussi des incertitudes quant à l'apport en contaminants sur quelques affluents, comme dans la campagne \#4 à proximité de LA03 avec le St-Michel (STM).

In fine, les différences générales entre le modèle et la mesure se situent dans la gamme 10 et 100 , ce qui est acceptable selon les diverses hypothèses formulées dans ce modèle et les incertitudes des mesures de concentration bactérienne.

\section{Exploitation du modèle mis en place}

\subsection{Analyse des conditions "normales"}

Afin de caractériser le comportement général de la qualité de l'eau dans l'estuaire, huit scénarios ont été élaborés en fonction du débit de la rivière, du flux des contaminants, de la température de l'eau et de l'amplitude des marées. Ces scénarios sont décrits dans le tableau 5. Ils ont été construits grâce aux nombreuses années de suivis.

Les résultats sont présentés dans le tableau 6 comme la concentration maximale atteinte pendant la simulation le long de l'estuaire pour les 8 scénarios simulés. 


\section{XVIèmes Journées Nationales Génie Côtier - Génie Civil \\ Le Havre 2020}

Tableau 5. Définition des 8 scénarios permettant de caractériser la qualité pour des "conditions normales" dans l'estuaire de la Laïta.

\begin{tabular}{lllll}
\hline $\begin{array}{l}\text { Conditions } \\
\text { hydrauliques }\end{array}$ & \multicolumn{2}{l}{$Q_{\text {Rivière hiver, } T_{\text {eau }}=9^{\circ} \mathrm{C}}$} & \multicolumn{2}{l}{$Q_{\text {Rivière été, }} T_{\text {eau }}=19^{\circ} \mathrm{C}$} \\
\hline $\begin{array}{l}\text { Flux de } \\
\text { contaminant }\end{array}$ & Période sèche & Période pluvieuse & Période sèche & $\begin{array}{l}\text { Période } \\
\text { pluvieuse }\end{array}$ \\
$\begin{array}{l}\text { Intensité } \\
\text { lumineuse }\end{array}$ & $\mathbf{I}_{0}=720000$ & $\mathbf{I}_{0}=\mathbf{2 0 0 0 0 0}$ & $\mathbf{I}_{0}=5 \mathbf{7 0 0 0 0 0}$ & $\mathbf{I}_{0}=\mathbf{1 0 0 0 0 0 0}$ \\
Morte eau & Scénario 1 & Scénario 3 & Scénario 5 & Scénario 7 \\
Vive eau & Scénario 2 & Scénario 4 & Scénario 6 & Scénario 8 \\
\hline
\end{tabular}

Tableau 6. Concentrations max. en E. Coli pour les 8 scénarios "conditions normales".

\begin{tabular}{ccccccccc}
\hline Scénario \# & LA12 & LA11 & LA10 & LA08 & LA07 & LA05 & LA03 & LA01 \\
\hline $\mathbf{1}$ & 500 & 754 & 550 & 371 & 260 & 246 & 105 & 25 \\
$\mathbf{2}$ & 501 & 862 & 571 & 398 & 254 & 245 & 100 & 26 \\
$\mathbf{3}$ & 1840 & 1821 & 1715 & 1556 & 1934 & 1455 & 1233 & 1031 \\
$\mathbf{4}$ & 1830 & 1826 & 1719 & 1558 & 1816 & 1492 & 1248 & 1064 \\
$\mathbf{5}$ & 686 & 544 & 71 & 56 & 318 & 83 & 2 & 2 \\
$\mathbf{6}$ & 666 & 520 & 87 & 61 & 318 & 108 & 16 & 1 \\
$\mathbf{7}$ & 1875 & 1713 & 1010 & 426 & 1762 & 417 & 160 & 78 \\
$\mathbf{8}$ & 1873 & 1690 & 1002 & 436 & 2152 & 1102 & 535 & 147 \\
\hline
\end{tabular}

Les concentrations les plus élevées d'E. Coli sont surtout en amont. En général, les concentrations changent fortement pendant le cycle des marées et surtout pour les débits "moyens" ou "faibles". Le taux de décomposition bactérienne dépend fortement du comportement du front de salinité.

Pour les débits "élevés" (l'hiver en période humide), les concentrations d'E. Coli sont relativement constantes de l'amont à l'embouchure de la rivière. L'estuaire est alors principalement composé d'eau douce le long de l'estuaire, indépendamment de la marée. Le taux de décomposition bactérienne est donc plus faible.

De manière générale, l'influence de la marée se fait ressentir uniquement sur les points en aval (de LA08 à LA01) et uniquement pour des débits fluviaux faibles.

\subsection{Analyse des rejets pics ou accidentels}

Les rejets dans les rivières et la concentration d'E. Coli présentés dans les 8 scénarios précédents sont fondés sur la médiane des mesures. D'autres scénarios ont également été étudiés avec des centiles 95 comme concentration maximale ou comme rejet accidentel. Un exemple est donné avec les scénarios 2 et 8 précédents qui sont simulés avec une concentration basée sur le centile 95 (7,106 UFC/100 ml pour STEP3 et 8,105 UFC/100 $\mathrm{ml}$ pour STEP2). Les résultats sont présentés dans le tableau 7 et sont comparés aux scénarios 2 et 8 . 


\section{Thème 6 - Gestion durable des zones littorales et estuariennes}

Tableau 7. Concentrations maximales en E. Coli pour les scénarios 9 et 10 comparés aux scénarios 2 et 8, échelle de couleur selon les concentrations et rapports.

\begin{tabular}{cccccccccc}
\hline & LA12 & LA11 & LA10 & LA08 & LA07 & LA05 & LA03 & LA01 \\
\hline Scénario 2 & 501 & 862 & 571 & 398 & 254 & 245 & 100 & 26 \\
Scénario 9 & 501 & 9263 & 4942 & 2925 & 1720 & 1739 & 633 & 122 \\
Rapport 9/2 & 1 & 11 & 9 & 7 & 7 & 7 & 6 & 5 \\
Scénario 8 & 1873 & 1690 & 1002 & 436 & 2152 & 1102 & 535 & 147 \\
Scénario 10 & 1873 & 8499 & 4079 & 1543 & 2182 & 1125 & 552 & 151 \\
Rapport 10/8 & 1 & 5 & 4 & 4 & 1 & 1 & 1 & 1 \\
\hline
\end{tabular}

Ces résultats montrent que pendant l'hiver en temps sec (scénarios 2 et 9), l'influence d'un pic de concentration au niveau des STEP 2 et 3 est visible jusqu'à LA01 alors qu'en été et par temps pluvieux (scénarios 8 et 10), elle est visible seulement jusqu'à LA07, à la confluence du Frout. En effet, la forte contamination de cet affluent lors des épisodes pluvieux a pour effet de masquer la contamination provenant des STEP 2 et 3.

Ces résultats permettent de confirmer l'impact des usines de traitement des eaux usées à l'embouchure des rivières pour des conditions particulières. Ce résultat est important car la zone de l'embouchure de la rivière concentre l'ensemble des activités d'élevage de moules et les problématiques de qualité des eaux de baignade. Par ailleurs le nautisme, même si pour cette activité il n'existe pas de norme particulière, est impacté sur tout le cours d'eau.

Cet exercice est effectué sur chaque source de contaminants afin de les hiérarchiser en s'appuyant sur une analyse multicritères (tableau 8). In fine, différentes solutions ont été proposées pour réduire la contamination des principales sources. Ces solutions comprennent par exemple, un traitement tertiaire pour les STEP, la suppression des abreuvements directs au cours d'eau, la création de bocage ou encore des travaux de réhabilitation sur le réseau d'assainissement collectif et individuel.

Toutes ces solutions ont également été testées avec un modèle numérique 3D afin d'apprécier les effets bénéfiques sur la qualité des eaux.

Tableau 8. Hiérarchisation des principales sources de contamination.

\begin{tabular}{lcccccccccc}
\hline Sources & $\begin{array}{c}\text { Isole } \\
\text { (IS2) }\end{array}$ & $\begin{array}{c}\text { Ellé } \\
\text { (EL2) }\end{array}$ & $\begin{array}{c}\text { Dourdu } \\
\text { (DOUR) }\end{array}$ & $\begin{array}{c}\text { STEP } \\
\mathbf{3}\end{array}$ & $\begin{array}{c}\text { STEP } \\
\mathbf{2}\end{array}$ & $\begin{array}{c}\text { Frout } \\
\text { (FR) }\end{array}$ & $\begin{array}{c}\text { Keryhuel } \\
\text { (KER) }\end{array}$ & $\begin{array}{c}\text { St Michel } \\
\text { (STM) }\end{array}$ & $\begin{array}{c}\text { Quinquis } \\
\text { (QUIN) }\end{array}$ & $\begin{array}{c}\text { STEP } \\
\mathbf{1}\end{array}$ \\
\hline Flux moyen & 2 & 1 & 3 & 4 & 5 & 7 & 8 & 9 & 6 & 10 \\
Flux pics & 2 & 1 & 4 & 5 & 7 & 3 & 9 & 8 & 6 & 10 \\
\hline
\end{tabular}

\section{Conclusion}

Pour conclure, un outil numérique a été mis en place pour représenter la dispersion bactérienne dans la rivière Laïta à partir d'une équation qui calcule le taux de désintégration d'E. coli en fonction de facteurs externes comme la salinité, l'afflux de 


\section{XVIèmes Journées Nationales Génie Côtier - Génie Civil \\ Le Havre 2020}

lumière et la température de l'eau. Le modèle a été calibré avec 4 campagnes in situ et donne une corrélation acceptable. L'étape d'étalonnage a montré l'importance de disposer d'un jeu complet de données et la nécessité de prendre en compte l'effet de la salinité dans un estuaire plutôt qu'un un taux de décroissance constant comme une loi T90 (temps nécessaires pour que $90 \%$ des bactéries meurent).

Une fois que l'outil numérique a été validé, une analyse complète de l'estuaire et des principales sources de contaminants a été effectuée sur la base de 23 simulations, pour évaluer les impacts sur les usages, hiérarchiser les sources et formuler des propositions d'actions correctrices.

\section{Références bibliographiques}

ACRI (2018). Etude hydrologique, hydraulique et hydro-sédimentaire de la Laïta amont. Rapport technique, réf. : A1523-1317-M-RE2-V1.1, Syndicat Mixte Ellé Isole Laïta SMEIL.

CHICK H., MARTIN C.J. (1908). The principles involved in the standardization of disinfectants and the influence of organic matter upon germicidal value. The Journal of Hygiene, Vol. 8(5), pp 654-697. https://doi.org/10.1017/s0022172400016016

DUGOR J., BAILLS J., RIHOUEY D. (2019). Numerical modelling of bacteriologic impacts in the Laïta estuary (France). XXVI ${ }^{\text {th }}$ Telemac \& Mascaret User Club Toulouse, 16-17 October, 2019, $6 \mathrm{p}$.

EGBERT G.D., EROFEEVA S.Y. (2002). Efficient inverse modeling of barotropic ocean tides. Journal of Atmospheric and Oceanic Technology, Vol. 19(2), pp 183-204. https://doi.org/10.1175/1520-0426(2002)019<0183:EIMOBO >2.0.CO;2

ERICHSEN A.C., DANNISØE J.G., JØRGENSEN C., MARK O., ANDKAAS H. (2006). Implementation and description of different early warning systems for bathing water quality, (Original title: Etablering af badevandsprofiler og varslingssystemer $i$ henhold til EU's nye badevandsdirektiv), Danish EPA, Miljøprojekt Nr 1101.

SELMEUS L. (2018). Dynamic modelling of bathing water quality with biodegradation of Escherichia coli in TELEMAC-3D. Master Thesis, TVVR 18/5001, Division of Water Resources Engineering, Department of Building \& Environmental Technology, Lund University, $74 \mathrm{p}$. 
Thème 6 - Gestion durable des zones littorales et estuariennes 